\title{
Variation in clinical usefulness of biomarkers of acute kidney injury in young children undergoing cardiac surgery
}

Hee Sun Baek, $\mathrm{MD}^{1}, *$, Youngok Lee, MD, $\mathrm{PhD}^{2, *}$, Hea Min Jang, $\mathrm{MD}^{1}$, Joonyong Cho, $\mathrm{MD}^{2}$, Myung Chul Hyun, MD, PhD ${ }^{1}$, Yeo Hyang Kim, MD, PhD ${ }^{1}$, Su-Kyeong Hwang, MD, PhD ${ }^{1}$, Min Hyun Cho, MD ${ }^{1}$

${ }^{1}$ Department of Pediatrics, Kyungpook National University, School of Medicine, Daegu, Korea; ${ }^{2}$ Department of Thoracic and Cardiovascular Surgery, Kyungpook National University, School of Medicine, Daegu, Korea

Background: Acute kidney injury (AKI) is one of the most significant postoperative complications of pediatric cardiac surgery. Because serum creatinine has limitations as a diagnostic marker of AKI, new biomarkers including neutrophil gelatinaseassociated lipocalin (NGAL), kidney injury molecule-1 (KIM-1), and interleukin-18 (IL-18) are being evaluated to overcome these limitations and detect AKI at an early stage after cardiac surgery.

Purpose: This study aimed to investigate the clinical usefulness of these biomarkers in young children.

Methods: Thirty patients with congenital heart diseases who underwent cardiac surgery using cardiopulmonary bypass (CPB) were selected, and their urine and blood samples were collected at baseline and 6, 24, and 48 hours after surgery. Serum creatinine and blood urea nitrogen levels as well as NGAL, KIM-1, and IL-18 levels in urine samples were measured, and clinical parameters were evaluated.

Results: Of the 30 patients, 12 developed AKI within 48 hours after cardiac surgery. In the AKI group, 8 of 12 (66.6\%) met AKI criteria after 24 hours, and urine KIM-1/creatinine (Cr) level (with adjustment of urine creatinine) peaked at 24 hours with significant difference from baseline level. Additionally, urine $\mathrm{KIM}-1 / \mathrm{Cr}$ level in the AKI group was significantly higher than in the non-AKI group at 6 hours. However, urine NGAL/Cr and IL-18/Cr levels showed no specific trend with time for 48 hours after cardiac surgery.

Conclusion: It is suggested that urine KIM-1/Cr concentration could be considered a good biomarker for early AKI prediction after open cardiac surgery using CPB in young children with congenital heart diseases.

Key words: Biomarkers, Acute kidney injury, Cardiac surgery, Child

\section{Key message}

Question: Can clinical usefulness of biomarkers of acute kidney injury vary on the clinical circumstances?

Finding: In young children undergoing cardiac surgery, urine KIM-1/Cr level peaked at 24 hours with significant difference from baseline level and was significantly higher at 6 hours in the AKI group. However, urine NGAL/Cr and IL-18/Cr levels showed no specific trend with time for 48 hours after cardiac surgery.

Meaning: Urine KIM-1/Cr concentration could be considered a good biomarker for early AKI prediction after open cardiac surgery in young children.

\section{Introduction}

Acute kidney injury (AKI) is one of the most significant postoperative problems of cardiac surgery using cardiopulmonary bypass $(\mathrm{CPB})$ among children with congenital heart disease, and its reported frequency varies from $20 \%$ to $50 \%$ depending on the definition of AKI used and the characteristics of the enrolled subjects. ${ }^{1-4)}$ AKI after cardiac surgery in pediatric patients is known to be linked with increased mortality and postoperative complications including a prolonged use of mechanical ventilation and the intensive care unit (ICU) stay. 5,6 )

AKI after cardiac surgery is usually evaluated by measuring urine output and serum creatinine level. However, serum creatinine has some limitations as a diagnostic marker of AKI because significant changes in serum creatinine level occur after $>50 \%$ reduction in renal function ${ }^{7,8)}$; additionally, serum creatinine concentration in children is affected by various factors including amount of muscle mass, volume distribution, and cardiac output, which especially vary among young children, ${ }^{9)}$ thereby ham. pering early recognition and intervention of AKI. ${ }^{10,11)}$

Recent studies have investigated novel AKI biomarkers to overcome the limitations of serum creatinine and detect AKI at an early stage after pediatric cardiac surgery. The clinical usefulness

\footnotetext{
Corresponding author: Min Hyun Cho, MD, Department of Pediatrics, Kyungpook National University Hospital, 130 Dongdeok-ro, Jung-gu, Daegu 41944, Korea 凶E-mail: chomh@knu.ac.kr, ORCID: https://orcid.org/0000-0002-7965-7587

*These authors contributed equally to this study as co-first authors.

Received: 9 August, 2019, Revised: 22 November, 2019, Accepted: 11 December, 2019

This is an open-access article distributed under the terms of the Creative Commons Attribution Non-Commercial License (http://creativecommons.org/licenses/by$\mathrm{nc} / 4.0 /$ ) which permits unrestricted non-commercial use, distribution, and reproduction in any medium, provided the original work is properly cited.

Copyright (c) 2020 by The Korean Pediatric Society
} 
of potential biomarker candidates such as neutrophil gelatinaseassociated lipocalin (NGAL), kidney injury molecule-1 (KIM-1), interleukin-18 (IL-18), and liver-type fatty acid-binding protein has been reported. ${ }^{7,9,12-14)}$ However, pediatric studies for AKI biomarkers have not produced definitive results in comparison with those from adult studies. This may be owing to the development of immunological and renal function in children as well as maturity of renal tubular function and structure being significantly different for different age groups, especially among young children.9)

The goal of this study was to investigate the clinical usefulness of these biomarkers for the early detection of AKI after cardiac surgery using $\mathrm{CPB}$ in young children with congenital heart diseases.

\section{Methods}

From November 2017 to October 2018, data were prospectively collected from 30 patients with congenital heart disease who had undergone cardiac surgery using CPB at Kyungpook National University Hospital. Patients aged $<18$ years at the time of surgery were included, and those with congenital anomalies of the kidney and urinary tract or renal insufficiency before surgery were excluded from the study. All patients were admitted before cardiac surgery and subjected to baseline blood tests and urinalysis. Urine $(5 \mathrm{~mL})$ and blood samples were collected from the patients during ICU stay at 6,24 , and 48 hours after cardiac surgery. After centrifugation of the urine samples at 3,000 rpm for 5 minutes, $2 \mathrm{~mL}$ of the supernatant was stored at $-80^{\circ} \mathrm{C}$ until use. Levels of serum creatinine using the isotope dilution mass spectrometry-traceable method and blood urea nitrogen as well as NGAL, KIM-1, and IL-18 levels in urine were measured. Clinical parameters including sex, age, weight, height, serum hemoglobin, echocardiography, type of congenital heart disease, preoperative percutaneous oxygen saturation $\left(\mathrm{SpO}_{2}\right)$ for cyanosis, presence of heart failure (Ross score $\geq 3$, which comprise of feeding volume consumed, time taken per feeding, respiratory rate, heart rate, respiratory pattern, peripheral perfusion, $\mathrm{S} 3$ or diastolic rumble, and hepatomegaly), ${ }^{15)}$ complexity of surgery (risk adjustment for congenital heart surgery-1 [RACHS-1] score), ${ }^{16)}$ duration of $\mathrm{CPB}$, aortic cross-clamp time, transfusion during surgery, and mean arterial pressure during $\mathrm{CPB}$ were also evaluated. The primary outcome variable was the development of AKI within 48 hours after surgery, and AKI was defined as $\geq 50 \%$ increase from baseline serum creatinine or urine output $<0.5 \mathrm{~mL} / \mathrm{kg} / \mathrm{hr}$ for 6-12 hours according to the Kidney Disease: Improving Global Outcomes guidelines. ${ }^{17)}$ The subjects were divided into 2 groups: AKI group included patients who met AKI criteria described above, and non-AKI group included patients who did not develop AKI.

Biomarker concentrations were measured using a commercialized enzyme-linked immunosorbent assay (ELISA) kit according to the manufacturer's instructions. Urine samples (100 $\mu \mathrm{L})$ or standards were incubated in microtiter plate wells that were precoated with a monoclonal antibody against human NGAL (Bioporto, Hellerup, Denmark), KIM-1 (R\&D Systems, Minneapolis, MN, USA), and IL-18 (MBL International, Woburn, MA, USA) for 1 hour at $37^{\circ} \mathrm{C}$. After liquid removal, each well was incubated with $100 \mu \mathrm{L}$ of biotinylated monoclonal antibody for 1 hour at $37^{\circ} \mathrm{C}$. After the solution was aspirated and the wells were washed 3 times, each well was incubated with $100 \mu \mathrm{L}$ of avidin-conjugated horseradish peroxidase for 1 hour at $37^{\circ} \mathrm{C}$. After the solution was aspirated and the wells were washed 5 times, the plates were incubated with $100 \mu \mathrm{L}$ of tetramethylbenzidine substrate solution for 10-30 minutes in the dark for color development; subsequently, absorbance was measured using an ELISA plate reader (Benchmark Plus, Bio-Rad, Hercules, CA, USA) at $450 \mathrm{~nm}$. Standard curves were constructed, and biomarker concentrations were presented as the mean values of 2 measurements. After NGAL, KIM-1, and IL-18 values at each time period were measured using ELISA, these values were adjusted for urine creatinine concentration.

Continuous variables were analyzed using Wilcoxon rank sum test, supposing relatively small size of sample data have nonparametric distribution. Categorical variables or proportions were compared using the chi-square test or Fisher exact test, as appropriate. For repeated measured analysis of nonparametric data, Friedman test with post hoc multiple comparison (Conover test) was used including Bonferroni correction. $P$ values less than 0.05 were considered statistically significant. All statistical analyses were performed using R ver. 3.4.3 (R Foundation for Statistical Computing, Vienna, Austria).

This study protocol and the informed consent documents were reviewed and approved by the Institutional Review Board of Kyungpook National University Hospital (IRB No. 2017-11033). Informed consent was obtained from all parents.

\section{Results}

Thirty patients were included in this study. The median patient age was 1.14 months (interquartile range [IQR], 0.68-3.61 months), and the male-to-female ratio was 2:1. The initial serum creatinine concentration was $0.24 \mathrm{mg} / \mathrm{dL}$ (IQR, $0.16-0.33 \mathrm{mg} /$ $\mathrm{dL})$. Seven patients (23.3\%) had heart failure, and 6 patients $(20 \%)$ showed cyanosis $\left(\mathrm{SpO}_{2}<85 \%\right)$. The most common congenital heart disease was ventricular septal defect (11 patients, $36.7 \%)$, followed by double-outlet right ventricle (4 patients, 13.3\%) (Table 1).

Of the 30 patients, 12 (40\%) developed AKI within 2 days after cardiac surgery using CPB. Table 2 shows a comparison of clinical features between the AKI and non-AKI group. Age, sex, and baseline characteristics including hemoglobin, mean arterial pressure, cardiac fractional shortening, ejection fraction, presence of heart failure, and the number of patients with $\mathrm{SpO}_{2}$ $<85 \%$ were not significantly different between the 2 groups. However, serum creatinine at baseline was significantly lower 
in the AKI group $(P=0.022)$. Most of the preoperative characteristics were not significantly different between the AKI and non-AKI groups. Although not quite significant, with regards to

Table 1. General characteristics of the study patients $(n=30)$

\begin{tabular}{lc} 
Characteristic & Value \\
\hline Age (mo) & $1.14(0.68-3.61)$ \\
Sex, male:female & $20: 10$ \\
Baseline serum creatinine (mg/dL) & $0.24(0.16-0.33)$ \\
No. of patients with heart failure & $7(23.3)$ \\
No. of patients with $\mathrm{Sp}_{2}<85 \%$ & $6(20)$ \\
Cardiac fractional shortening (\%) & $36.95(32.98-38.10)$ \\
Ejection fraction (\%) & $70(64.35-71.20)$ \\
Type of heart disease & \\
Ventricular septal defect & $11(36.7)$ \\
Double-outlet right ventricle & $4(13.3)$ \\
Atrial septal defect & $3(10)$ \\
Transposition of the great arteries & $2(6.7)$ \\
Mitral regurgitation & $2(6.7)$ \\
Tetralogy of Fallot & $2(6.7)$ \\
Total anomalous pulmonary venous return & $2(6.7)$ \\
Aortic stenosis & $1(3.3)$ \\
Interrupted aortic arch & $1(3.3)$ \\
Coarctation of the aorta & $1(3.3)$ \\
Atrioventricular septal defect & $1(3.3)$ \\
No. of patients who developed AKI & $12(40)$ \\
\hline
\end{tabular}

Values are presented as median (interquartile range) or number (\%). $\mathrm{SpO}_{2}$, percutaneous oxygen saturation; $\mathrm{AKI}$, acute kidney injury.
CPB time, marginally significant difference was shown between the 2 groups $(P=0.059)$. None of the study subjects died or needed dialysis. Finally, all patients with AKI recovered fully.

Serum creatinine and urinary biomarker concentrations with and without adjustment for urine creatinine at each time point after cardiac surgery in the AKI group are shown in Table 3, including the number of patients met AKI criteria. The number of patients who met AKI criteria was 4 (33.3\%), 5 (41.7\%), and 3 (25.0\%) for 6, 24, and 48 hours after surgery, respectively. After repeated measured analysis, it was found that serum creatinine level in the AKI group increased significantly at 24 hours, and 48 hours ( $P=0.0105$ and $P=0.0084$, respectively) compared to the baseline level. Urine KIM-1 level showed a tendency to increase until 24 hours, and then decrease. Furthermore, KIM-1/Cr (with adjustment of urine creatinine) showed significant increase at 24 hours from baseline level $(P=0.0017)$. In contrast, urine NGAL, NGAL/Cr, IL-18, and IL-18/Cr levels showed no specific trend with time until 48 hours after surgery.

Because of their nonparametric distribution, serum creatinine and urinary biomarker concentrations with adjustment of urine creatinine in both AKI and non-AKI group at each time point are presented in a box plot format (Fig. 1). Except for serum creatinine concentrations at baseline (AKI group: 0.160 [IQR, 0.143-0.245], non-AKI group: 0.280 [IQR, 0.220-0.370]; $P=0.022$ ), most of the measurements in the AKI group were similar to or higher than those in the non-AKI group at each time point. Especially, urine KIM-1/Cr level at 6 hours after cardiac surgery was significantly higher in the AKI group than in the non-

Table 2. Comparison of clinical characteristics between the AKI and non-AKI groups

\begin{tabular}{|c|c|c|c|}
\hline Characteristic & Non-AKI $(n=18)$ & $\operatorname{AKI}(n=12)$ & $P$ value \\
\hline Age (mo) & $1.26(0.72-12.16)$ & $1.06(0.54-3.04)$ & 0.799 \\
\hline Male sex & $11(61.11)$ & $9(75.00)$ & 0.693 \\
\hline \multicolumn{4}{|l|}{ Baseline characteristics } \\
\hline Hemoglobin (g/dL) & $12.4(11.3-14.0)$ & $12.4(11.1-14.25)$ & 0.983 \\
\hline Serum creatinine (mg/dL) & $0.28(0.22-0.38)$ & $0.16(0.14-0.25)$ & $0.022^{*}$ \\
\hline Mean arterial pressure $(\mathrm{mmHg})$ & $59.0(50.0-67.0)$ & $53.0(50.0-60.0)$ & 0.431 \\
\hline Cardiac fractional shortening (\%) & $36.9(32.9-38.0)$ & $37.0(34.5-38.2)$ & 0.850 \\
\hline Ejection fraction (\%) & $70.0(64.6-71.0)$ & $70.0(64.5-71.4)$ & 0.760 \\
\hline Presence of heart failure (\%) & $4(22.22)$ & $3(25.0)$ & 1.000 \\
\hline $\mathrm{SpO}_{2}<85 \%(\%)$ & $2(11.11)$ & $4(33.33)$ & 0.305 \\
\hline \multicolumn{4}{|l|}{ Operative characteristics } \\
\hline CPB mean arterial pressure $(\mathrm{mmHg})$ & $45.0(41.5-50.0)$ & $45.0(40.0-55.0)$ & 0.882 \\
\hline CPB time (min) & $58.0(48.5-91.5)$ & $83.5(76.5-135.5)$ & 0.059 \\
\hline Cross-clamp time (min) & $39.0(30.0-59.5)$ & $57.0(44.0-70.0)$ & 0.172 \\
\hline Use of transfusion (\%) & $10(55.56)$ & $8(66.67)$ & 0.819 \\
\hline RACHS-1 score & & & 0.335 \\
\hline 1 & $4(22.22)$ & $0(0)$ & \\
\hline 2 & $9(50.00)$ & $7(58.33)$ & \\
\hline 3 & $3(16.67)$ & $3(25.00)$ & \\
\hline 4 & $1(5.56)$ & $2(16.67)$ & \\
\hline 5 & $1(5.56)$ & $0(0)$ & \\
\hline
\end{tabular}

Values are presented as median (interquartile range) or number (\%).

AKI, acute kidney injury; $\mathrm{SpO}$ 2, percutaneous oxygen saturation; CPB, cardiopulmonary bypass; RACHS-1, risk adjustment for congenital heart surgery-1.

*statistical significance $P<0.05$ 
Table 3. Concentrations of serum creatinine and urinary biomarkers with and without adjustment for urine creatinine at each time point after cardiac surgery in the AKI group $(n=12)$, including the number of patients met AKI criteria $(\geq 50 \%$ increase in the serum creatinine level)

\begin{tabular}{lcccc}
\hline Variable & 0 Hour & 6 Hours & 24 Hours & 48 Hours \\
\hline S-Cr (mg/dL) & $0.160(0.143-0.245)$ & $0.245(0.183-0.335)$ & $0.345 *(0.245-0.493)$ & $0.355^{*}(0.313-0.410)$ \\
AKI onset (\%) & - & $4(33.3)$ & $5(41.7)$ & $3(25.0)$ \\
NGAL $(\mathrm{ng} / \mathrm{dL})$ & $402.991(161.343-552.009)$ & $356.886(152.741-554.192)$ & $275.868(137.139-441.564)$ & $257.216(155.216-658.852)$ \\
$\mathrm{NGAL} / \mathrm{Cr}(\mathrm{ng} / \mathrm{mg})$ & $34.143(9.920-46.782)$ & $21.701(8.998-27.840)$ & $33.157(22.406-43.653)$ & $40.380(7.222-77.131)$ \\
$\mathrm{KIM}-1$ (ng/dL) & $26.663(2.097-48.109)$ & $126.274(77.062-162.728)$ & $195.617(137.189-323.250)$ & $62.626(34.593-125.018)$ \\
$\mathrm{KIM}-1 / \mathrm{Cr}(\mathrm{ng} / \mathrm{mg})$ & $2.956(0.546-4.538)$ & $6.532(4,768-10.259)$ & $15.171 * *(13.676-23.912)$ & $3.488(2.286-13.437)$ \\
$\mathrm{IL}-18(\mathrm{ng} / \mathrm{dL})$ & $6.913(4.370-19.736)$ & $17.399(7.107-37.543)$ & $6.495(1.454-12.991)$ & $5.366(0.835-15.477)$ \\
$\mathrm{IL}-18 / \mathrm{Cr}(\mathrm{ng} / \mathrm{mg})$ & $0.578(0.214-3.760)$ & $0.842(0.339-1.960)$ & $0.462(0.073-6.501)$ & $0.152(0.093-1.131)$ \\
\hline
\end{tabular}

Values are presented as median (interquartile range) or number (\%).

AKI, acute kidney injury; S-Cr, serum creatinine; NGAL, neutrophil gelatinase-associated lipocalin; KIM-1, kidney injury molecule-1; IL-18, interleukin-18; Cr, urine creatinine.

*Statistical significance, $P<0.05$ when compared to its baseline ( 0 hour) level. ${ }^{* *} P<0.01$ when compared to its baseline level.
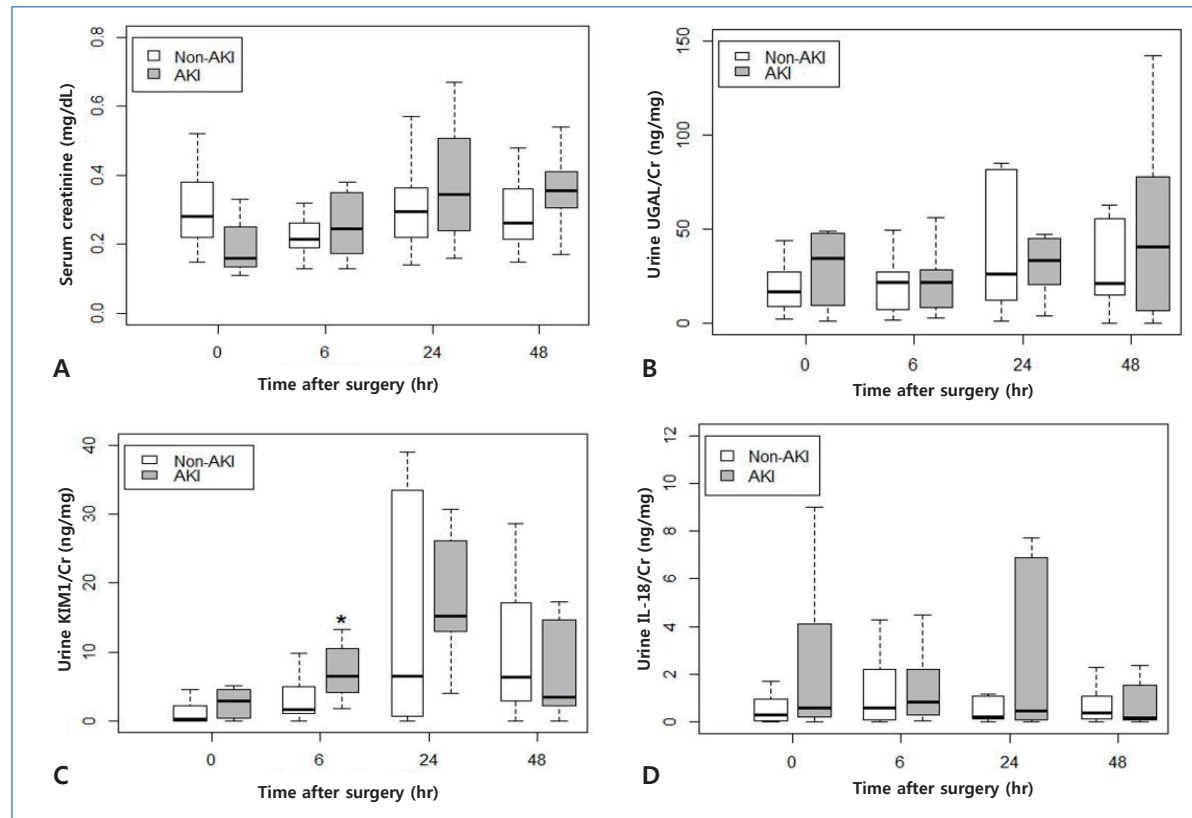

Fig. 1. Comparison of serum creatinine and urinary biomarker concentrations with adjustment for urine creatinine between the acute kidney injury (AKI) and non-AKI groups at each time point after cardiac surgery. The box ranges from the first (Q1) to the third quartile (Q3) of the distribution and represents the interquartile range (IQR). Box plot with whiskers extends to $\pm 1.5 \times \mid \mathrm{QR}$. A line across the box indicates the median. ${ }^{*}$ The AKI group showed a significantly higher $\mathrm{KIM}-1 / \mathrm{Cr}$ concentration at 6 hours after surgery than the non-AKI group $(P=0.003)$.

AKI group (AKI group: 6.543 [IQR, 4.768-10.259], non-AKI group: 1.705 [IQR, 1.056-4.953]; $P=0.003$ ). As seen in Fig. 1, $\mathrm{KIM}-1 / \mathrm{Cr}$ peaked at 24 after surgery, this finding is consistent with the data reported in previous studies. $\left.{ }^{7}, 18\right)$ However, there were not significant difference between AKI and non-AKI group at any time point, with regards to concentrations of urine NGAL/ Cr $(P=0.586, P=0.767$, and $P=0.614$ for 6,24 , and 48 hours, respectively) and urine IL-18/Cr $(P=0.556, P=0.873$, and $P=$ 0.614 for 6,24 , and 48 hours, respectively). Furthermore, the temporal trend of NGAL/Cr and IL-18/Cr concentration in the present study was not consistent with that reported in previous studies.

\section{Discussion}

Cardiac surgery with CPB is used to treat children with congenital heart diseases. However, reduced perfusion and nonpulsatile renal blood flow during $\mathrm{CPB}$ cause hypoxic-ischemic renal injury, leading to tubular and endothelial cell injury. ${ }^{7,19)}$ The incidence of AKI after cardiac surgery using CPB in children is reportedly as high as 50\% and is related to younger age, singleventricle status, higher RACHS-1 category, higher baseline serum creatinine level, and longer CPB time. ${ }^{20,21)}$ However, in this study, both the AKI and non-AKI group did not show significantly different characteristics except for baseline serum creatinine.

Postoperative AKI causes an increase in the length of ICU stay and use of intensive treatments such as mechanical ventilation 
and continuous renal replacement therapy. Furthermore, children with postoperative AKI are more likely to progress to endstage renal disease and are more prone to the hazard of mortality. ${ }^{22)}$ Therefore, early diagnosis and continuous follow-up of these patients are recommended. For the early recognition of AKI, several biomarkers have been introduced in the recent decades to overcome the challenges posed by traditional methods based on serum creatinine level and urine output.

Although many cohort studies have been conducted to date to determine effective AKI biomarkers, no definitive results have been produced in pediatric studies as opposed to adult studies. This may be owing to the maturity of renal tubular function and structure being significantly different across different age group.9) Especially among young infants, the cutoff range of urine AKI biomarkers is known to be significantly different from individuals of other age groups because the renal tubules are immature to function appropriately and cannot optimally concentrate urine. However, few studies have been conducted to identify the age-specific differences in children under the age of $18 .{ }^{9,23)}$ Fortunately, because most of the enrolled patients in this study were young infants (median age: 1.14 months old), we could minimize the age-dependent discrepancy for the cutoff value of biomarkers as opposed to previous studies.

NGAL is the most widely studied biomarker of AKI after cardiac surgery in children. This protein is recognized to be highly upregulated in renal proximal tubule cells owing to ischemic injury. Previous studies have reported a more than 10 -fold increase in urine NGAL levels after $\mathrm{CPB} ;{ }^{7,8,18,24)}$ however, in this study, urine NGAL levels did not change for 48 hours after surgery. We carefully suppose that this result might be owing to the young age of our subjects and delayed sampling time. The younger the child when $\mathrm{CPB}$ is performed, the earlier AKI will develop after CPB. Additionally, because urine NGAL level usually begins to increase at 2 hours after $\mathrm{CPB}$, reaches a peak at 6 hours, and subsequently decreases, we suppose that urine sampling at 6 hours after CPB, performed as in our study, was not suitable for detecting a significant elevation in urine NGAL level.

KIM-1 production is low in a normal kidney, whereas its upregulation and production significantly increase in renal proximal tubule cells owing to ischemic injury. ${ }^{25)} \mathrm{Han}$ et al. ${ }^{26)}$ reported that urine KIM-1 concentration increased at 6-12 hours and continued to exist at a high concentration for 48 hours after $\mathrm{CPB}$; they suggested that urine KIM-1 concentration at 12 hours after cardiac surgery was a prognostic factor for AKI in children with an area under the curve (AUC) value of 0.83 . In the present study, urine KIM-1/Cr concentration started to increase at 6 hours and peaked at 24 hours showing significant difference from baseline level $(P=0.0017)$, which is consistent with previous studies. In particular, the level of urine KIM-1/Cr at 6 hours after cardiac surgery using $\mathrm{CPB}$ was significantly higher in the AKI group $(P=0.003)$ than in the non-AKI group. In the present study, AKI developed after 24 hours after surgery in majority of the AKI group (8 of 12). Therefore, we suggest that urine KIM-1 could be considered a good biomarker for early AKI predictions related with cardiac surgery.

Concentration of IL-18, a proinflammatory cytokine produced in the renal proximal tubule, reportedly increases at $6 \mathrm{~h}$ and peaks at 12 hours after $\mathrm{CPB} .{ }^{7,27}$ Parikh et al. suggested that an increased urine IL-18 level during the first 6-12 hours after $\mathrm{CPB}$ was a moderate predictor for postoperative $\mathrm{AKI}$ in patients with AUC values of $0.72-0.82 .{ }^{28)}$ In the present study, urine IL18 and IL-18/Cr level showed no specific trend.

This study had several limitations. First, because this was a single-center study, the patient sample size was relatively smaller than that in previous studies. Second, it was difficult to precisely evaluate the increase and decrease in its concentrations because the biomarker measurement interval considered in this study was not optimally spaced. The measurement should have been performed at an earlier time after surgery to evaluate changes in concentrations of biomarkers, which rapidly increase in urine. Third, urine creatinine adjustment may not be accurate as adult or older children due to very young age of patients in this study. Urine creatinine is an index that reflects the amount of muscle, which is lower in children than in adults. Especially, among young infants with immature kidneys to produce optimally concentrated urine, the urine creatinine value may be substantially lower" ${ }^{9}$; therefore, its significance as an adjustment factor may not be high. Lastly, postoperative parameters such as the length of ICU stay and use of mechanical ventilation were not examined.

Nevertheless, the strength of this study is that subjects were mostly infants within 3 months of age (median, 1.14; IQR, 0.683.61). Because studies on these young infants are very scarce, this study could help find effective biomarkers for AKI prediction that could be applied to these age groups.

In summary, it is suggested that urine $\mathrm{KIM}-1 / \mathrm{Cr}$ concentration could be considered a good biomarker for early AKI prediction after open cardiac surgery using CPB in young children with congenital heart diseases. Furthermore, continuous follow-up of pediatric patients with AKI and subclinical AKI (increase in biomarker levels but not in serum creatinine levels) after cardiac surgery is required.

\section{Conflicts of interest}

No potential conflict of interest relevant to this article was reported.

\section{Acknowledgments}

This work was supported by Biomedical Research Institute grant, Kyungpook National University Hospital (2016).

\section{References}

1. Li S, Krawczeski CD, Zappitelli M, Devarajan P, Thiessen-Philbrook $\mathrm{H}$, Coca SG, et al. Incidence, risk factors, and outcomes of acute kidney injury after pediatric cardiac surgery: a prospective multicenter study. Crit Care Med 2011;39:1493-9.

2. Hirano D, Ito A, Yamada A, Kakegawa D, Miwa S, Umeda C, et al. Independent risk factors and 2-year outcomes of acute kidney injury after 
surgery for congenital heart disease. Am J Nephrol 2017;466:204-9.

3. Lex DJ, Tóth R, Cserép Z, Alexander SI, Breuer T, Sápi E, et al. A comparison of the systems for the identification of postoperative acute kidney injury in pediatric cardiac patients. Ann Thorac Surg 2014;97:202-10.

4. Park SK, Hur M, Kim E, Kim WH, Park JB, Kim Y, et al. Risk factors for acute kidney injury after congenital cardiac surgery in infants and children: a retrospective observational study. PLoS One 2016;11:e0166328.

5. Tóth R, Breuer T, Cserép Z, Lex D, Fazekas L, Sápi E, et al. Acute kidney injury is associated with higher morbidity and resource utilization in pediatric patients undergoing heart surgery. Ann Thorac Surg 2012;93: 1984-90.

6. Gil-Ruiz Gil-Esparza MA, Alcaraz Romero AJ, Romero Otero A, Gil Villanueva N, Sanavia Morán E, Rodríguez Sánchez de la Blanca A, et al. Prognostic relevance of early AKI according to pRIFLE criteria in children undergoing cardiac surgery. Pediatr Nephrol 2014;29:1265-72.

7. Jefferies JL, Devarajan P. Early detection of acute kidney injury after pediatric cardiac surgery. Prog Pediatr Cardiol 2016;41:9-16.

8. Mishra J, Dent C, Tarabishi R, Mitsnefes MM, Ma Q, Kelly C, et al. Neutrophil gelatinase-associated lipocalin (NGAL) as a biomarker for acute renal injury after cardiac surgery. Lancet 2005;365:1231-8.

9. Greenberg JH, Parikh CR. Biomarkers for diagnosis and prognosis of AKI in children: one size does not fit all. Clin J Am Soc Nephrol 2017;12: 1551-7.

10. Hwang YJ, Hyun MC, Choi BS, Chun SY, Cho MH. Acute kidney injury after using contrast during cardiac catheterization in children with heart disease. J Korean Med Sci 2014;29:1102-7.

11. Park JT. Postoperative acute kidney injury. Korean J Anesthesiol 2017;70: 258-66.

12. Toda Y, Sugimoto K. AKI after pediatric cardiac surgery for congenital heart diseases-recent developments in diagnostic criteria and early diagnosis by biomarkers. J Intensive Care 2017;5:49.

13. Malhotra R, Siew ED. Biomarkers for the early detection and prognosis of acute kidney injury. Clin J Am Soc Nephrol 2017;12:149-73.

14. Dong L, Ma Q, Bennett M, Devarajan P. Urinary biomarkers of cell cycle arrest are delayed predictors of acute kidney injury after pediatric cardiopulmonary bypass. Pediatr Nephrol 2017;32:2351-60.
15. Ross RD, Bollinger RO, Pinsky WW. Grading the severity of congestive heart failure in infants. Pediatr Cardiol 1992;13:72-5.

16. Jenkins KJ, Gauvreau K, Newburger JW, Spray TL, Moller JH, Iezzoni LI. Consensus-based method for risk adjustment for surgery for congenital heart disease. J Thorac Cardiovasc Surg 2002;123:110-8.

17. Section 2: AKI definition. Kidney Int Suppl 2012;2:19-36.

18. Yuan SM. Acute kidney injury after pediatric cardiac surgery. Pediatr Neonatol 2019;60:3-11.

19. Devarajan P. Update on mechanisms of ischemic acute kidney injury. J Am Soc Nephrol 2006;17:1503-20.

20. Aydin SI, Seiden HS, Blaufox AD, Parnell VA, Choudhury T, Punnoose A, et al. Acute kidney injury after surgery for congenital heart disease. Ann Thorac Surg 2012;94:1589-95.

21. Blinder JJ, Goldstein SL, Lee VV, Baycroft A, Fraser CD, Nelson D, et al. Congenital heart surgery in infants: effects of acute kidney injury on outcomes. J Thorac Cardiovasc Surg 2012;143:368-74.

22. Gist KM, Kwiatkowski DM, Cooper DS. Acute kidney injury in congenital heart disease. Curr Opin Cardiol 2018;33:101-7.

23. Libório AB, Branco KM, Torres de Melo Bezerra C. Acute kidney injury in neonates: from urine output to new biomarkers. Biomed Res Int 2014; 2014:601568.

24. Devarajan P. Neutrophil gelatinase-associated lipocalin--an emerging troponin for kidney injury. Nephrol Dial Transplant 2008;23:3737-43.

25. Al-Ismaili Z, Palijan A, Zappitelli M. Biomarkers of acute kidney injury in children: discovery, evaluation, and clinical application. Pediatr Nephrol 2011;26:29-40.

26. Han WK, Waikar SS, Johnson A, Betensky RA, Dent CL, Devarajan P, et al. Urinary biomarkers in the early diagnosis of acute kidney injury. Kidney Int 2008;73:863-9.

27. Parikh CR, Mishra J, Thiessen-Philbrook H, Dursun B, Ma Q, Kelly C, et al. Urinary IL-18 is an early predictive biomarker of acute kidney injury after cardiac surgery. Kidney Int 2006;70:199-203.

28. Parikh CR, Coca SG, Thiessen-Philbrook H, Shlipak MG, Koyner JL, Wang Z, et al. Postoperative biomarkers predict acute kidney injury and poor outcomes after adult cardiac surgery. J Am Soc Nephrol 2011;22: 1748-57. 